\title{
Giovanni Ceva ed il suo carteggio inedito con Vincenzio Viviani.
}

\author{
(Di Alberto Pascal, a Napoli.)
}

\begin{abstract}
1. $\mathbf{\mathrm { Z }}$ ben conosciuto il nome di Grovann Geva, matematico milanese del secolo XVII. Egli appartenne a quella scuola di matematici che s'era andata formando in Milano verso la fine di quel secolo, e della quale fecero parte Tommaso Crva (1648-1737), che gli era fratello, Girolamo Sagcheri (1667-1733), Pifetro Paolo Garavagaro senior (1617-1688) e Piftro Paolo Garavagaro junior (1659-1723), e, senza dubbio, altri che pur forse meriterebbero d'esser tratti dall'oblio.

Uno studio comparativo dei progressi che questa scuola apportò alle scienze esatte, sarebbe oggi veramente desiderabile; uno studio condotto sul tipo di quello che il prof. LonIa ci diede a proposito della Scuola napoletana di Nicola Fergora $\left(^{1}\right)$. Già ho dato il mio piccolo contributo agli studi che su questa scuola di matematici potrebbero compiersi, tentando di illustrare la vita e l'opera matematica del maggior rappresentante di essa, GIrolamo Saccheri $\left({ }^{2}\right)$, e ricordando l'apparecchio che, nel 1695 , ideò Tommaso Ceva per la plurisezione degli angoli $\left({ }^{3}\right)$.
\end{abstract}

2. Ma, dopo Groolamo Sacchen, ci appare la figura di Giovann Ceva come quella di un geometra veramente eminente. Egli rimase pressochè di-

(1) G. Loria, Nicola Fergola e la scuola di Matematici che lo ebbe a duce (Atti della Regia Università di Genova, pubblicati per le onoranze a Cristoforo Colombo, 1892).

(') A. PAScal, Girolamo Saccheri nella vita e nelle opere (Giornale di Matematiche di Battaglini, (3) vol. 52, 1914); Sopra una lettera inedita di Girolamo Saccheri (Atti del R. Istituto Veneto di Scienze, Lettere ed Arti, t. 74, 1914-15).

${ }^{(3)}$ A. PascaL, L'apparechio polisettore di Tommaso Ceva ed una lettera inedita di Guirlo Grandi (Rendiconti del R. Istituto Lombardo di Scienze e Lettere, vol. XLVIII, 1915).

Annali di Matematica, Serie III, Tomo XXIV. 
menticato, sino a quando lo CHasles, nel suo Aperçu historique ( $\left.{ }^{4}\right)$, non fece notare tutta la copia di risultati a cui il Geva era giunto nella sua opera: De lineis rectis se invicem secantibus statica constructio $\left({ }^{5}\right)$. Lo CHASLES prima, e poi il Cremona $\left({ }^{6}\right)$, fecero vedere come, per primo, Grovanni Ceva avesse avuto l'idea di servirsi delle proprietà del centro di gravità d'un sistema di punti nella teoria delle trasversali, dove cioè occorre considerare rapporti di segmenti determinati dai punti di intersezione di rette.

Quando si suppongono connessi in questi punti, pesi inversamente proporzionali ai segmenti staccati, potrà dedursi, dal rapporto di questi pesi, il rapporto fra i segmenti. Seguendo quest'ordine di idee, Ceva dimostra il notissimo teorema che oggi da lui prende il nome; lo dimostra prima col suo metodo statico; poi ne riporta due altre dimostrazioni geometriche che egli dice dovute all'amico suo Pietro Paolo Caravaggio (junior). Da quel teorema, egli ricava numerose proprietà e teoremi, e, fra gli altri, CEva deduce anche il cosidetto teorema di Tolomeo; poi, sempre col suo metodo, giunge a dimostrare anche parecchie delle proprietà conosciute del triangolo; e, proseguendo ancora, dimostra proprietà sul tetraedro, sul quadrilatero gobbo, ecc.; ed, infine, nel libro secondo, dimostra anche che in un poligono circoscritto ad una conica, $i$ punti di contatto dividono $i$ lati del poligono in segmenti tali che il prodotto di quelli non aventi estremi a comune, è uguale al prodotto dei rimanenti.

Come ha fatto notare Cremona, il Corrocis ( ${ }^{7}$ ) pervenne, nel 1811, a gran parte dei teoremi di Ceva, senza conoscere il libro di questi: egli diede nove teoremi, dei quali alcuni non son compresi nell'opera di CEva, e gli altri comprendono, come casi particolari, i teoremi che vi sono contenuti.

Questa opera di GEva, per la quale egli può giustamente considerarsi come il precursore delle idee fondamentali che MöBIus applicò per creare il

( $\left.{ }^{4}\right)$ M. Chasles, Apèrçu historique sur l'origine et le développement des méthodes en Géométrie, particulièrement de celles qui se rapportent à la Géométrie moderne, etc., II édit. (Paris, Gauthier-Villars, 1875), Note VII, pp. 294-296.

(5) G. GEVA, De lineis rectis se invicem secantibus statica constructio (Mediolani, ex typ. Ludovici Montiae, MDGLXXVIII).

(') L. Gremona, Intorno ad un'operetta di Giovanni Ceva matematico milanese del secolo XVII (Rivista gimnasiale e delle Scuole tecniche e reali, t. VT, 1859; Opere matematiche, Milano, Hoepli, 1914, t. I, p. 208).

(7) In parechi lavori riassunti in Coniouss, Sur la théorie des momens considerée comme analyse des rencontres des lignes droites (Journal de l'École Polytechnique, cahier 24, 1835). 
suo Calcolo baricentrico, fu, come dicevamo, sempre dimenticata; il ConıoLs, in nota della sua Memoria citata, avvertiva che sol quando già aveva finito il suo lavoro, si accorse della esistenza del libro del geometra milanese. Essa è menzionata appena nell' Histoire des mathématiques del Montucla $\left(^{8}\right)$, il quale avvertì peraltro che solo conosceva il nome del Ceva per gli accenni che di questi, e delle sue opere, aveva falto Guido Grand nel suo libro sulla logaritmica $\left({ }^{9}\right)$.

3. Quasi nessuna notizia ci è stata tramandata purtroppo sopra Grovanni Crva: un suo stesso contemporaneo, l'Argelat, nel redigere l'articolo destinatogli nella sua Bibliotheca Scriptorum Mediolanensium $\left({ }^{10}\right)$, ci avverte di aver tentato inutilmente di ricercar notizie sopra Grovanni Ceva a Mantova (dove cioè Geva, secondo ogni probabilità, dovette morire); e si limita perciò a dar l'elenco (alquanto scorretto) delle opere di lui, e qualche scarsa notizia.

L'anno della nascita ci è ignoto; egli appartenne ad una famiglia di ricchi banchieri di Milano; suo padre si chiamava Garlo Francesco, e sua madre Paola Colomba. Tutti i fratelli di Giovanni si diedero alla religione; il maggiore di età, Nrcolò, fu carmelitano scalzo; un altro, Gristoroko, fu gesuita, e fu poeta di merito; l'altro fratello fu Tommaso, ancor egli gesuita e celebre poeta e matematico $\left({ }^{11}\right)$.

Grovanni fu Commissario della Camera Arciducale del Ducato di Mantova; e purtroppo null'altro ci è dato, per ora, di aggiungere alle notizie

(8) J. E. Montucla, Histoire des Mathématiques dans laquelle on rend compte de leurs progrès depuis leur origine jusqu'à nos jours; etc. Tome II (Paris, chez Jombert, MDCCLVIII), p. 72.

${ }^{9}$ ) Nella Epistola geometrica ad virum clarissimum Thomam Cevam Societatis Jesu, riportata in fine dell'opera G. GRANDI, Geometrica denonstratio Theorematum Hugenianorim circa Logisticam, seu logarithmicam lineam, etc. (Florentiae, MDCGI, apud Brigonci).

(10) Ph. Argelatr, Bibliotheca Seriptorum Mediolanensium seu Acta, et Elogia virorum omnigenaeruditione illustrium, qui in Metropoli insubriae oppidisque circumjacentibus orti sunt; etc. Tomus primus (Mediolani, in Aedibus Palatinis, 1745), parte 2. ${ }^{\mathrm{a}}$, col. 417.

(ii) G. G. Cordara, Vita del P. Tomaso Ceva lin Le vite degli Arcadi illustri seritte da diversi autori, e pubblicate d'ordine della Generale Adunanza da Michel GiUseppe MoRE, Custode d'Arcadia, parte V (Roma, tip. De' Rossi, 1751)]. - Prolusione agli studi nel solenne aprimento del Liceo di Milano in Porta Nuova recitata il giomo 24 novenbre 1812 da CESARE Rovida profesonre di Matematica, e reggente provvisionale (Milano, dalla stamperia Pirotta, 1813), pag. 45. 
sulla sua vita, se non che studiò matematica sotto la guida del livornese Donato Rossettu, come Ceva stesso ci dice nel proemio al De lineis rectis $\left({ }^{12}\right)$.

Dall'elenco delle sue opere, riportato dal RicGard $\left({ }^{13}\right)$, appare che il Cuva, oltre che di pura matematica s'occupò anche specialmente di questioni d'idraulica, e anche del problema della moneta, scrivendo al riguardo un'opera De re numaria $\left({ }^{14}\right)$.

('2) Quando questo nostro lavoro era già stato ultimato, il chiarissimo prof. Gino Loria è riuscito a corrispondere in parte alle speranze nostre sul ritrovamento di notizie circa la vita di Giovann Ceva. Egli ha resi noti $\mathrm{i}$ risultati suoi in una breve nota: Per la biografia di Giovanni Ceva (Rendiconti del R. Istituto Lombardo di Scienze e Lettere, vol. XLVIII, 1915).

Nell'Archivio di Stato di Mantova (Archivio Gonzaga) esistono alcune buste con scritti vari di Ceva, e di lui si parla inoltre in altri documenti in quell'Archivio custoditi: da tutta questa messe preziosa di documenti si potrebbe perciò ricavare parte della biografia di Giovanni Ceva.

Altre notizie su Ceva, il Loria riproduce dall'opera inedita di Carlo d'Arco, intitolata Famiglie mantovane; dalla quale appare, tra l'altro, esser Giovanni Geva nato nel dicembre 1647, e morto nel 1734 .

$\left(^{13}\right)$ Biblioteca Matematica Italiana dalla origine della stampa ai primi anni del secolo XIX compilata dal dott. ing. PEetro Riccard (Modena, tip. Soliani, MDCCCLXX), col. 342-343.

(4) Crediamo opportuno riportare la lista delle opere di Grovana Ceva:

1) De lineis reetis se invicem secantibus statica constructio (Mediolani, ex typ. Montiae MDCLXXVIII).

2) Opuscula Mathematica de potentiis obliquis, de pendulis, de vasis, et de fluminibus (Mediolani, ex typ. Montiae, 1682).

3) Geometria motus. Opusculum geometricum a JoANNE CEVA mediolanensi in gratiann Aquarum excogitatum. Continet duos libros. Primum de simplici motu, alterum de composito (Bononiae, typ. Pisarij, MDCXGII).

4) Tria problemata geometris proposita, una cum ipsius ratiocinio in gravitatem omnigeni corporis ostendendam (Mantuae, ex typ. Pazzoni, 1710).

5) De re numaria quoad fiori potuit geometrice toactata, ecc. (Mantuae, apud Pazzonum, MDCGXI).

6) De mundi fabrica, unico yravitatis principio innixa, deque fluminibus, ecc. (Mantuae, 1715).

7) Le conseguenze del Reno, se coll'aderire al proggetto de' Signari Bolognest, si permettesse in Pò grande (In Mantova, per Pazzoni, MDCCXVI).

8) Replica di Grovann Ceva in difesa delle sue dimostrazioni, e ragioni, per le quali non debbasi introdurre Reno in Pò, contro la risposta datasi dal signor dottor Eustachio Man fredi (In Mautova, per Pazzoni, MDCGXVII).

9) Risposta di Glovann Ceva alle osservazioni del signor dottor Eustachio Manfredi contro la di lui replica in proposito dell'immissione di Reno in Pò grande, pretesa da' signoni Bolognesi (In Mantova, per Pazzoni, MDCCXXI).

10) Opus hydrostaticum (Mantuae, apud Pazzonum, MDCCXXVIII). 
4. Visto che sulla vita di questo illustre geometra così poco oggci si conosce, può senza dubbio offrire un qualche interesse la notizia del ritrovamento di molte sue lettere, nell'epistolario dell' «ultimo discepolo di GaIflei » Vrnaenzio Vivian, che si custodisce nella R. Biblioteca Nazionale di Firenze. L'importanza eccezionale, per la storia della matematica del secolo XVII, che offre il carteggio del Vivianı, è stata additata agli studiosi dall'illustre storico della scuola galileiana, Antonıo Favaro $\left({ }^{\text {is}}\right)$.

Nulla, credo, per la storia scientitica degli ultimi secoli, ha maggiore importanza delle lettere che ci son conservate nelle Biblioteche e negli Archivi; in quelle lettere che fra loro si trasmettevano i dotti del tempo, essi si comunicavano tutte le Joro nuove ricerche, i risultati che via via ottenevan negli studi loro: lettere che, sebbene non destinate alla pubblicazione, facevano in certo modo per quei tempi l'ufficio delle moderne Note e comunicazioni accademiche. Del resto la importanza che in alcuni casi potevan avere queste comunicazioni fu riconosciuta da essi stessi: e cosi, per esempio, vediamo Waltis, Coldrss, Nawton, Leibniz, Frnmat, ecc., raceglier gram parte delle lettere ch'essi s'eran andati scambiando, per stampare j Commercia epistolica, così importanti per la storia dei progressi, specialmente dell'analisi, nei secoli XVII-XVIII.

Il carteggio di VIncenzıo VIvian è conservato, assieme ai manoscritti del medesimo, nei rolumi 142-148 dei Manoscritti Galileiani, Discepoli, della Biblioteca Nazionale di Firenze. Tutti questi manoscritti di VIvianı furon lasciati dal Vivian in eredità all'abate Jacopo Panzanin, morto il quale furon posti in vendita, e gran parte di essi potè esser recuperata ed acquistata da G. B. Glemente Nelis. Di tutti questi manoscritti, il prof. Favaro ha dato un utilissimo elenco alla fine della citata sua biografia del Vivianı; ed ha dato altresì un elenco dei corrispondenti di questi, quale risulta dal carteggio.

Per comprendere in modo adeguato tutta la importanza che potrebbe avere la pubblicazione del carteggio di Vivianr, bisogna pensare alla grande fama che raggiunse, ai suoi tempi, questo scienziato, e per merito dei suoi lavori geometrici, e, specialmente fra questi, della sua divinazione del $V$ libro delle Coniche di Apollonio $\left({ }^{16}\right)$.

$\left({ }^{15}\right)$ Cfr. A. FAVAro, Amici e corrispondenti di Galileo Galilei, XXIX, Vincenzio Viviani (Atti del R. Istituto Veneto di Scienze, Lettere ed Arti, t. 72, 1912-13).

(16) V. VIVIANI, De maximis et minimis geometvica divinatio in quintun Conicorum Apollonit Perguei adhuc desideratum, ecc. (Florentiae, apud 1. Cocchini, MDCLIX). Per la storia particolareggiata di questa divinazione di Apollonio, si vegga A. Favaro, loc. eit. 
Tutti i matematici più illustri dell'epoca avevan comunicazioni col Vrvians, e, fra i nomi de'suoi corrispondenti, troviamo quelli di Leibn17, HuYgens, Wallis, de l'Hospital, Varignon, ecc., per non far cenno che degli stranieri. Son molte poi anche le copie delle lettere di risposta del Vivian, molto spesso però redatte in caratteri illeggibili, e senza nome di destinatario, nè data.

Antonio Favaro è l'unico che abbia estratto qualcosa da questo prezioso carteggio $\left({ }^{17}\right)$; vadano a questo illustre Maestro, che in Italia tiene cosi alto l'onore degli studi di storia delle scienze, i miei più vivi ringraziamenti per gli aiuti ed i consigli preziosi dei quali m'è stato largo.

5. Le lettere che abbiamo trovate sono le seguenti:

I. G. Geva a Viviani; 5 ottobre 1685 (tomo 145, car. 236).

II. Vrviani a G. Geva; 16 ottobre 1685 (tomo 146, car. 285 t).

III. G. Geva a Viviani; 26 ottobre 1685 (tomo 146, car. 286).

IV. Viviani a G. Ceva; 11 novembre (?) 1685 (tomo 143, car. 70).

V. G. Geva a Vivians; 23 novembre 1685 (tomo 146, car. 287).

VI. G. Ceva a Vivianr; 17 gennaio 1686 (tomo 147, car. 1).

VII. G. Geva a Viviani; 8 febbrajo 1686 (tomo 147, car. 2).

VIII. G. Ceva a Viviani; 20 febbrajo 1693 (tomo 145, car. 286).

IX. Vrviani a G. Ceva; 3 marzo 1693 (tomo 143, car. 125).

X. G. Ceva a Viviani; 13 marzo 1693 (tomo 147, car. 125).

XI. G. Ceva a Viviani; 11 marzo 1701 (tomo 147, car. 193) $\left({ }^{18}\right)$.

6. Dalla prima lettera che riproduciamo, si scorge che Ceva cominciò sin dal 1685 ad occuparsi delle ricerche ch'egli poi raccolse nel suo libro, pubblicato sette anni dopo:

(17) A. FAvAro, Due lettere inedite del $F$. Gerolamo Succheri a. C. a. G. a Vincenzio Viviani (Rivista di Fisica, Matematica e Scienze Naturali, anno IV, 1903); Contribuzioni inedite al carteggio di Evangelista Torricelli. (Bollettino di Bibliografia e Storia delle Scienze Matematiche, anno xVI, 1914).

${ }^{(19)}$ La brevissima lettera VI non la riprodurremo, perchè ei è sembrata di nessuna importanza.

Ringrazio pubblicamente il distinto signor Mario Montagna che, con vero spirito di abnegazione e di amicizia, trasse per me, dalla Biblioteca di Firenze, copia dei documenti riportati in questo lavoro. 
Geometria motus. Opusculum geometricum à IoAnñ Geva mediolanensi in gratiam aquarum excogitatum. Continet duos libros. Primum de simplici motu, alterum de composito (Bononiae, M.DG.XGII, typ. Pisarij).

Liesempio che Ceva comunica a Vivian è contenuto nella prop. XII, theor. XII del volume.

Assieme alla lettera di Ceva abbiamo anche la copia della risposta del VIVIANI; ecco ambo le lettere:

$$
\text { Ill." Sig.", Sig." Pne Col. }{ }^{m o} \text {, }
$$

È un secolo che non ho riverito con mie linee il sig." Vincenzo mio S.", il di cui merto serbo sempre avanti gli occhi. Nel tempo che son stato fuori in villa ho impiegato qualche hore in veder se mi riesciva alcuna inventione nell'argomento dell'aque, et ho havuto quella fortuna, che non speravo dalla mia inabilità; con essa occasione mi è riuscito farmi largo campo nella scienza de' moti, e loro tempi; et è methodo così universale, e geometrico, che serve per ritrovare cose geometriche, ciò̀ a quadrare de' curvilinei; le darò qui un esempio, senza la dimostrazione per non estendermi con un processo.

Sia la curva di tal sorte $B C D$ \& che tirate fra essa, e la retta $A G$ le perpendicolari $A B, E C, F D$ alla stessa $A G ;$ sia sempre $F D$ ad $A B$ in proportion sottodoppia di $A G$ a $G \vec{F}$; e cosi $E C$ ad $A B$ in proportion sudupplicata di $A G$ a $G E$. È noto a V. S. Ill. ${ }^{\text {ma }}$ che la perpendicolare $G \&$ è assintoto, ciò̀ a dire non concorrerà mai colla curva $B C D \& ; s i$ chè il piano \&B $A G \&$ sarà senza fine longo alla parte \&. Hora io dico che questo piano è uguale al doppio rettangolo di $G A$ in $A B$, e che

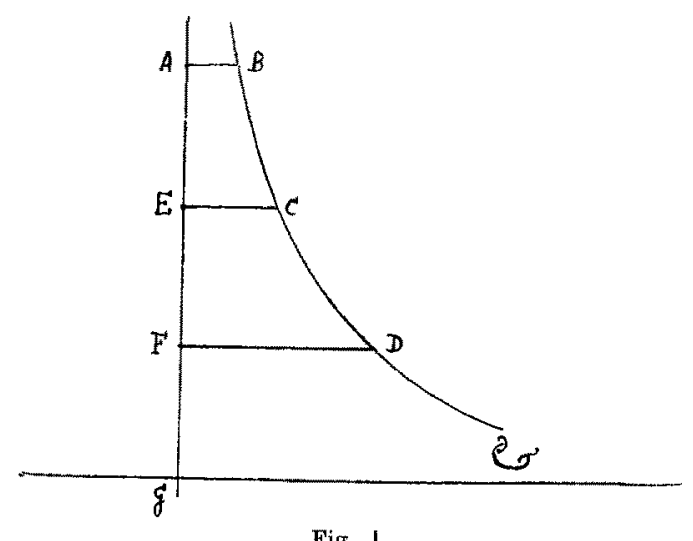

Fig. 1. qualsisia portione $B D F A$ tra le ugualm..$^{t \theta}$ distanti $A B, F D$ è uguale al rettangolo di $A B$ nella doppia differenza di $A G$ sopra la media proportionale tra $A G, e G F$.

Ma sia hora la detta curva di tal natura, che $l a$ FD alla $A B$ stia in 
proportion triplicata della suduplicata di $A G$ a $G F$, e così $E C$ ad $A B$ in proporzion triplicata di $A G$ alla media proporzionale tra $A G, e G E$.

Qui havrei di bisogno che mi fosse indicato da V.S. Ill. ${ }^{\text {ma }}$ qualche autore dal quale potessi trovare artificio di quadrare qualche delle portioni $A C$, $E D$ etc., tra le sue paralelle; giachè per altro il curvilineo $\& B A G \&$ si dimostra infinito per grandezza; io non ne suplico direttivam. ${ }^{t e}$ V. S. Illma, perchè sarebbe troppo mio ardire attese le occupat. ${ }^{n i}$ gravi di V. S. Ill." med..

Io mi ho affaticato un pezzo ma indarno per esser cosa che molto mi preme per le dimostrat. ${ }^{n i}$ dell'aque; e che senza questa, mi conviene, o lasciar molto, o veram. ${ }^{\text {to }}$ restar sospeso.

Onde la priego con tutto lo spirito a farmene l'honore richiesto; e mentre la supplico conservarmi vivo nella sua pregiatis. ${ }^{\text {ma }}$ grazia, resto con farle hum. ${ }^{\text {ma }}$ riverenza. Mant. ${ }^{a}$ li 5 ott. 85.

di V. S. Ill."'

$$
\text { devot."m" et obligat." Ser. }
$$

Grovanni Cena.

addì 16 ottobre

1685 al Sig. Gio. Ceva

Mantova.

$$
\text { Ill. }{ }^{\text {mo }} \text { Sig." Pad. } .^{\circ} \text { Col." }{ }^{m \prime \prime}
$$

Parmi, se io non erro, la prima delle due curve linee indicatemi da V. S. Ill. ${ }^{\text {ma }}$ con la favorit. ${ }^{m a}$ sua del 5 del corrente esser la stessa di quella la quale in fine del mio Diporto Geometrico pubblicato nel 1676 in quel Trattatello de Modi varj Meccanici, Lineari e Solidi per la bisezzione dell'angolo e per le due medie proporzionali $\left(^{19}\right)$ io chianai Iperbole Mesolabica e la quale

(19) Si allude al libretto sui Modi vari meccamici, lineari, e solidi tentati da V. V. per le construzioni de'due illustri problemi, il primo, della divisione dell'angolo in data proporzione, il secondo, dell'invenzione delle due medie proporzionali, contenuto nel Quinto libro degli Elementi di Euclide, ovvero Scienaa miversale delle proporzioni spiegata colla dottrina del Galileo, con mov'ordine distesa, e per la prima volta mebblicata da Vixcenzio Viviani, ultimo suo discepolo. Agginutevi cose varie, e del Galileo, e del Torricelli; i ragguauli dolle ultime. opere loro, com altro, che dall'indice si mrnifesta (In Firenze, alla Condotta, MDCLXXIV). a pag. 979 del quale si fa parola della iperbole mesolabica. 
dissi aver raffigurata per la s.t" $^{\text {th }}$ delle innumerabili Iperbole, cioè per quella in cui le abscisse da una delle due Asintote prese dal Centro, o concorso delle med." Asintote stanno in proporzion reciproca dell'ordinata $q .^{\text {'a }}$ applicate alle med. asintote e la qual curva io conclusi quivi per ultimo esser ella peraltro maravigliosa, intendendo io per cagione dell'esser quadrabile quello spazio benchè d'estensione infinita, si come le sue parti; e per aver anche in se altre ammirabili proprielà.

La generatione poi dell'altra curva di V. S. Ill. ${ }^{\text {na }}$, s'io pur non mi inganno, si riduce ad esser la stessa che quella, nella quale $i$ Cubi delle dette abscisse, stanno in proporzione reciproca de' quadrati delle d: applicate, e la. quadratura dello spazio di questa che è immensurabile da una parte, ma ben mensurabile dall'altra la troverò $V$. S. Ill." enunciata con altre innumerabili.... del Commercio Epistolico scritto da Mons." Fermat Francese al Caval." Digbi Inglese $\left({ }^{20}\right)$.

Tanto penso io che basta per soddisfare ad desid. ${ }^{\circ}$ e comanda:" $d i V$. S. Ill." la quale per i gran progressi che io mi prometto dal di lei acutissimo ingegno, esorto con.... esultanza, a coltivare adesso che ell'è sul verde degli anni suoi, queste nobili scienze, affinchè non le avvenga ciò che è avvenuto a me, che da 40 e più anni in quà mi sono immerso in affari tanto remoti da queste speculazioni che ormai despero di poterle pì ripigliare per farle pubbliche, come ne vengo stimolato da più parti.

E qui per fine etc.

Di V.S. Ill. ${ }^{m a}$

$$
\begin{aligned}
& \text { Dev. }{ }^{m o} \text { Oblig. }{ }^{m o} \text { Ser. } \\
& \text { VINGENZO VIVIANI. }
\end{aligned}
$$

(') Commercium epistolicum, de quastionibus quibusdam Mathematicis nuper habitum. Inter nobilissimos viros D. Gulielnum Vicecomitem Brouncker, anglum; D. Kenelmum Digby, item Equitem anglum; D. Fermatium, in suprema Tholosatum Curia Iudicen primarium; D. Freniclum, nobilem parisinum. Una cum D. Joh. Wallis Geomet. Profess. Oxonii. D. Franc. a Schooten, Math. Prof. Lugduni Batavorum; aliisque. Edidit Johannes WALLis, S. Th. D. in celeberrima Oxoniensi Academia Geometriae Professor Savilianus. (Oxonii, Excudebat A. Lichfield, Acad. Typograph. Impensis Tho. Robinson, M.DC.LVIII).

[La copia da me consultata è della Biblioteca Vittorio Emanuele di Roma, e vi è segnato a mano «Ex lib. Alph. Borelli»].

Questo libro fu tradotto in francese e riprodotto per intero nel vol. III delle Oeuvres de Fermat publiées par les soins de MM. Pavl Tannery et Chanles Henry sous les auspices du Ministère de l'Instruction Publique (Paris, Gauthier-Villars, MDCGCXCVI), pp. 399-610. 
La iperbole mesolabica alla quale accenna Vincenzio Viviani nella precedente lettera, fu da lui descritta per la costruzione delle due medie proporzionali; egli prima l'aveva

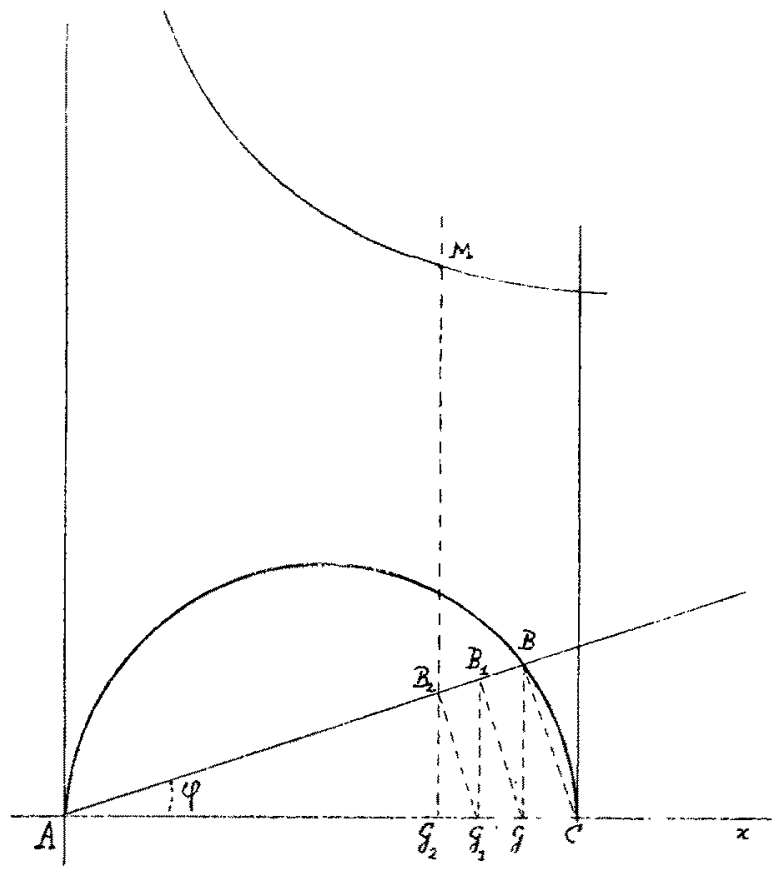

Fig. II. chiamata cissoide mesolabica (pel modo col quale l'otteneva, alquanto analogo a quello con cui si ottiene la cissoide di Diocle dal cerchio); ma si accorse poi esser quella curva la medesima di quella che altri (come diremo ora) aveva già considerato.

Questa curva non è che un caso particolare della curva che si ottiene come segue $\left({ }^{21}\right)$ : si consideri (vedi figura II) un semicerchio di diametro $A C=a$, e, per $A$, si conduca una retta arbitraria che tagli la circonferenza in $B$, ed in $D$ la tangente alla circonferenza condotta nel punto $C$. Costruiamo la $B G$

perpendicolare ad $A C$, la $G B_{1}$ perpendicolare ad $A D$, la $B_{1} G_{1}$ perpendicolare ad $A C$, la $G_{1} B_{2}$ perpendicolare ad $A D$, e così via.

Sull'ultima retta così ottenuta $B_{k-1} G_{k-1}$ si stacchi un segmento $G M=A D$; il luogo del punto $M$ (al variare, naturalmente, della retta condotta per $A$ ) è una curva (che può chiamarsi iperbole) d'ordine $2 k+1$, la cui equazione è :

$$
x y^{2 k}=a^{2 k+1} \text {. }
$$

Invero, detto $\varphi$ l'angolo $D A C$, ed $(x, y)$ le coordinate di $M$, si ha:

$$
y=A D=\frac{a}{\cos \varphi}
$$

(21) G. LoRIA, Spezielle algebraische und transzendente ebene Kurven. Theorie und Geschichte. Deutsche Ausgabe von F. Sarüтte. II Auflage. Erster Band (Leipzig und Berlin, Tenbner, 1910), p. 319. 


$$
\begin{array}{rlrl}
A B & =a \cos \varphi & A G=a \cos ^{2} \varphi \\
A B_{1} & =a \cos ^{3} \varphi & A G_{1} & =a \cos ^{4} \varphi \\
\ldots \ldots \ldots \ldots & \ldots \ldots \ldots \\
A B_{k-1} & =a \cos ^{2 k-1} \varphi & A G_{k-1} & =x=a \cos ^{2 k} \varphi
\end{array}
$$

eliminando $\varphi$ dalla prima ed ultima relazione, si ottiene, per equazione del luogo di $M$ :

$$
x y^{2 k}=a^{3 k+1} \text {. }
$$

Per $k=1$ la costruzione precedente si riduce a quella della curva del terzo ordine:

$$
x y^{2}=a^{3}
$$

che è quella che Vivian I chiama iperbole mesolabica. Questa medesima curva è trattata da Fenмat nella lettera IV del Commercium citato; nella lettera cioè, del 20 aprile 1657 , a DugbY $(1603-1665)\left({ }^{22}\right) ;$ a proposito di essa, Fermat enuncia la stessa proprietà notata da G. Ceva nella lettera da noi riportata; nella iperbole ordinaria il rettangolo $N P$ è costante (v. figura III); si supponga ora (dice Fermat) che sia costante il prodotto del quadrato di $B N$, per

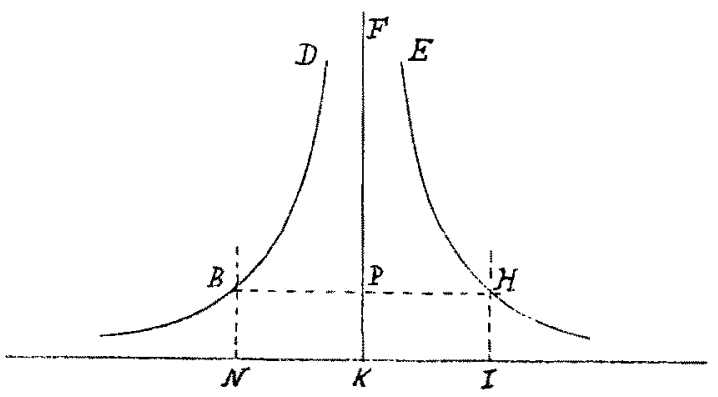

Fig. III. $B P$, che cioè, quindi, come diremmo noi, l'equazione della curva, riferita agli assintoti, sia $x y^{2}=$ cost. La curva allora sarà una iperbole tale che il parallelogrammo $B I$ sarà eguale allo spazio compreso fra la base $B H$ e le due curve $B D F, F E H$.

Se, invece, è costante il prodotto del cubo di $B N$ per $B P$, si otterrà un'altra iperbole, tale che il parallelogrammo $B I$ sarà doppio dello spazio compreso fra la base $B H$ e le due curve. E così poi, in generale, se il prodotto d'una potenza di $B N$ per una potenza di $B P$ è costante, il parallelogrammo $B I$ starà allo spazio suddetto, come la differenza fra l'esponente

(2x) E riprodotta come lettera LXXXII della corrispondenza di Fermat, stampata nel volume II delle Oeuvres de FERMAT già citate. 
della potenza di $B N$ e l'esponente della potenza di $B P$, sta all'esponente della potenza di $B P$.

Ne segue, osserva Fenмat, che nell'ordinaria iperbole lo spazio suddetto non è eguale ad uno spazio dato, e che, in generale, l'esponente di $B N$ deve esser maggiore di quello di $B P$.

Le stesse considerazioni aveva fatte prima WALLIS nella sua Arithmetica infinitorum $\left({ }^{23}\right.$ ) (prop. 102 e 104 ), come scrive lo stesso Wallis al Diabr (lettera V del Commercium).

7. All'ultima lettera riportata Ceva rispondeva ancora:

$$
\text { Ill." Sig.", Sig." Prne Col." }
$$

La favorit." ${ }^{\text {ma }}$ di V.S. Ill." mi è sembrata un tesoro, e per le grazie che mi fa, e per le notizie massime che m'accenna. Quà in Mantova non si sa dove ricorrere per un libro in genere di Mathematica, onde ho scritto al Pre Ceva Gesuita mio fr.ello in Milano che esso pure si diletta di simili scienze, anzi le professa in Brera, doppo il pranzo, havendo impiegata la matina nell'insegnar la Rettorica $\left({ }^{24}\right)$; l'ho dico pregato a far diligenza in biblioteca Ambrosiana, acciochè mi rimetta la coppia di quella lettera contenuta nell'opera che mi accenna, con insieme il titolo preciso, osia la notitia d'haver il medesimo libro del Valisio. Ma ho anche premura, et ansietà grande di godere dell'opera di V. S. Ill." ${ }^{m a}$, che pur m'accenna, eperò se è cosa piccola V. S. Ill. ${ }^{m a}$ è supplicata, e sententiata a rimettermela subbito. Mi par di vedervi cose bellissime, et anco utilissime al mio proposito sichè Le rinovo l'istanza; inoltre so che son. da niente, e che parla in me solo l'affetto grande, che porto a simili scienze, ardisco come scolaro miserabile verso un suo gran Maestro di solecitarla a trovar tempo per dar alla luce il parto che mette in dubbio, nè permetta in

${ }^{(23)}$ Johannis WaLlisil SS. Th. D. Geometriae Professoris Saviliani in celeberrimá Academia Oxoniensi Arithmetica infinitorum sive Nova methodus inquirendi in Curvilineorum quadraturam aliaque difficiliora Matheseos Problemata (Oxonii, Typis Leon. Lichfield Academiae Typographi, Impensis Tho. Robinson, Anno 165̆6).

(24) Il p. Tommaso Ceva, gesuita, insegnò nel Collegio di Brera in Milano, per ben 45 anni, la Matematica. 
modo alcuno, che si sepelliscano nel niente speculationi sue, che per la grandezza loro, e chiarezza sono ammirate da tutto il Mondo. Assicuro V. S. Ill."' che io oggidi non stimo quasi altro Geometra, che la persona di V. S. Ill." med.", "nè ho dubbio che io non m'habbia a cavar grandem." la sete, a per meglio dire riacenderla piì per la qualità troppo sapovita del liquore e vena sua geometrica. Conoseo d'essermi avvanzato troppo, però la suplico con un riflesso della sua gran bontà compative la mia penna mentre con non più diffundermi..... sempre qual fui

Di V.S. Ill." Mant. ${ }^{\text {ma }}$ li 26 ott. 85.

$$
\text { Devot." }{ }^{m o} \text { et obligat.". Ser. }
$$

Grovanni Cieva.

8. Ed ecco poi come Vivian signorilmente annunzia al Ceva la sua intenzione di donargli il Commercium che egli non poteva trovare in Mantova:

\author{
Al Sig. Gio. Ceva \\ Mantova \\ $11 \ldots . .1685$
}

\title{
Ill. ${ }^{\text {no }}$ Sig." mio Prne Col."
}

L'amore intenso che ho e che di continuo ho avuto alle Matematiche è stato di tal tempra e così staccato dall'amor di me stesso che quantunque io per la magg. parte del tempo da che io ne ho qualche cognizione, io mi sia trovato in impieghi.... dal poter applicare a dar fuori le cose nie, ho però sempre (t tutto mio potere cooperato che gli altri vi si adoperino, e si facciano onore col promuoverle con le lor nuove speculazioni. Di qui è che ora vedendo V. S. Ill." ${ }^{\text {ma }}$ tutta intenta e in ordine a farci godere le sue, e sentendo all'incontro per la di lei amorevol."'" del $22\left({ }^{* 5}\right)$ del passato che le manchi quel Commercio Epist." del Vallisio d'onde io le scrissi che poteva cavarne notizie al bisogno.....e dopo molte dilig. falleci non l'avendo trovato mi son risoluto di buon quore (sic) a privarmene con staccarlo da altre opere colle quali era legato, e di farvene un libero dono insieme con le mie bagatelle stampate che mi dice non aver ancor vedute e che mi ha comandato inviarle.

(25) Dovrebbe dire 26. 
Il tutto la supplicherei per sua benignità a voler gradire in segno della venerazione che ho al merito suo, e del desid. ${ }^{\circ}$ ardent. ${ }^{m o}$ delle sue glorie. Resta che si compiaccia d'indicarmi per qual via io debba farle pervenire questi libri, e a chi io debba qua consegnarle ad effetto che le giunghino sicuri e ben condizionati. Mentre non essendo questa per altro [la copia della lettera finisce così senz'altro].

[manca la firma].

E Geva così risponde ringraziando:

$$
\text { Ill. }{ }^{\text {mo }} \text { Sig. }{ }^{*} \text { Sig. mio Pne Col. }{ }^{\text {mo }}
$$

Quando cominciavo quasi a temere della mia importunità verso di V.S. Ill.".$^{\text {ma }}$ con forse essermi abbusato delle sue grazie, ecco che son oltremodo favorito dalla gentilissima sua, che sol hora ricevo; dalla quale per le sovrabbondanti grazie che mi significa esser V.S. Ill. ${ }^{m a}$ per farmi, colla missione delle sue nobili speculationi, et altro, concepisco di goder senza fallo la fortuna d'esser tra' privilegiati servi di V. S. Ill." ${ }^{\text {ma }}$; e già chè ha così disposto di graziarmi potrà restar anco servita di far avere il tulto à Sig. ${ }^{\prime i}$ Simon, e Flaminio Piazza, che questi rimettendolo con prime occasioni a questi mercanti ebrei Franchetti, da essi ricupererò ogni cosa spero bene condizionata; però la suplico quanto so, e posso a non volere per causa mia guastare il complesso di quelle sue opere che mi dice, con estrarvi il comercio epistolico, che non mi ̀̀ mai riuscito di poterlo far rinvenire, ne meno nella Biblioteca Ambrosiana $\left({ }^{26}\right)$ da dove pure mancano poche opere, che son di proffitto al Mondo letterario, che se poi è cosa già seguita, mi riuscirà ben favore segnalato. Non so propriamente svilupparmi in mostrarle colla penna que' segni che esprimano le mie molte obligazioni, so bensi di conservarle nell'anima sempre vive, sicuro di non poterle smaltire, se V. S. Ill." non me ne dà in qualche parte la forma, con nuovi favori, ciò̀ con qualche materia per ubidirla. Resto dunque con uniliarmi a' suoi cenni, rassegnandomi sempre di V.S. Ill. ${ }^{\text {ma }}$

Mant. ${ }^{a}$ li 23 nov. 1685

$$
\text { dev. }{ }^{\text {no }} \text { et obligat." Ser. }{ }^{\text {re }}
$$

\section{Giovanni Ceva.}

(28) Oggi neppure esiste alla Biblioteca Ambrosiana di Milano il libro del WaLLis; come pure non esiste alla Biblioteca Nazionale della medesima città, alla quale il fondo di libri antichi di matematica pervenne dalla Biblioteca dell'ex-Collegio di Brera della Compagnia di Gesù. 
9. L'ultima lettera, infine, di questo periodo di tempo, è la seguente :

$$
\text { Ill." Sig.", Sig. }{ }^{*} \text { Prne Col. }{ }^{\text {mo }}
$$

Hebbi alla venuta del corriere nell'ordinario scorso $i$ tre favoritissimi libri $\left({ }^{27}\right)$, con la gentilissima di V.S. Ill. ${ }^{m a}$, e perchè il dono, massime da una mano così riverita, e che di doni n'è la compositrice, non mi poteva riuscire più prezioso, perciò ne devo a V.S. Ill." , dovuti miglioni (sic) di grazie. Non ho potuto a meno di non riccorrere l'ultima opera di V.S. Ill. ${ }^{\text {ma }}$ in qualche parte, riserbandomi di vederla attentam..$^{\text {te }}$ piacendomi assaissimo il methodo del dimostrare il quinto libro delle proporzioni d'Euclide mettendo per comuni notizie, quelle cose che per dimostrarle, ha dovuto Euclide supporre cose molle più demostrabili; mi piaciono poi assaissimo le dimostr." della trisetl." dell'angolo, e delle due medie. In quanto poi al bisogno mio per hora, ho osservato la proposit." generale nella lettera $2^{a}$ in francese del Caval." Digbi, circa q." piani di longhezza infinita, e loro quadratura; ma la dimostratione non mi è riuscita di vederla nelle seguenti lettere. In ogni caso quando mai altro, mi basterò che la proportione assegnata di d.' quadrature sia infallibile, e che mi sia approvata da V. S. Ill."m

Non posso per hora maggiormente diffundermi, onde carico di molte obligazioni resto sempre Mantova 8 febr. 1686.

Di V. S. Ill. ${ }^{m x}$

$$
\text { Devot. }^{\text {mo }} \text { et obligat." }{ }^{\text {"mo }} \text { Sre }
$$

Grovanni Geva.

\section{S. Vincenzo Viviani Firenze.}

10. Uscito, verso la fine del 1692, stampato a Bologna, il libro Geometria motus del Cera, questi ne inviò copia al Viviani, colla lettera che segue, a proposito della quale notiamo che la dimostrazione del Viviani che Ceva dice di aver riportata nel suo libro, è quella di p. 55 della Geometria motus.

(27) I tre libri inviati al Cava dovettero essere il Commercium di Wallis, il Quinto libro degli elementi di Euclide del VIVIANI, già citato, e l'altra opera di VIVIANI stesso: De maximis et minimis geometrica divinatio in quintum Conicorum Apollonii Pergaei adhuc desideratum, ete. (Florentiae, typ. Cocchini, MDCLIX), nella quale, come si è detto, Viviani divinò il $\mathrm{V}$ libro di Apollonio. 


\section{Ill." Sig." mio S. Prne Col."no}

$\grave{E}$ un gran tempo che non ho con mie riverito V.S. Ill. ${ }^{m a}$, la di cui eccellente virti mi sta sempre viva nanti gli occhi, e ne ho havuto un grande esemplare legendo il gran bel libro di V.S. Ill. ${ }^{\text {ma }}$ de maximis et minimis $\left({ }^{28}\right)$ dove si vede la miniera fecondissina del di lei grande ingegno. Ho rossore in mandargli un mio ultimo libriciuolo inscritto geonetria motus, e mi si accresce tanto piu quanto che temo esser la miglior parte di quello le dimostr. ${ }^{n i}$ che la mi mandò già gran tempo fa de' pendoli per diffesa del celeberimo G. Gallilei, anzi dissi della verità. La supplico a compatire questo ardire, $e$ se non altro riconoscere nel povero mio libro l'ossequio che le porto. Mi son inoltre preso libertà d'inviargli due altre coppie, acciochè l'una la faccia tenere al S. D." Lorenzo Bellini d." leggente in Pisa, et altra al S. Ant." Magliabecchi $\left({ }^{29}\right)$ ambi miei SS."i riverili, e supplicandola a compassionare le mie debolezze, e scusare $i$ disturbi che le aggimng, resto di V. S. Ill."

Mant." li 20 febr. 1693 .

Devot." ${ }^{\text {mo }}$ et obligat. ${ }^{\text {mo }} S^{\text {re }}$

Giovanni Ceva.

A questa Viviani rispose colla lettera che segue, che porta, certo per errore, la data del 1692; la copia di questa lettera, di mano del Vivianr, presenta in parechi punti cancellature e sgorbi, ed è scritta in modo che, in qualche punto riesce indecifrabile, od almeno, se pur si comprendono le singole parole, non si comprende il senso complessivo della frase: ond'è che io ho creduto miglior cosa sostituire dei puntini in luogo dei passi e delle parole suddette.

${ }^{29}$ ) È l'opera (inviata dá Vivianı a Ceva) nella quale Viviani compì la sua famosa divimazione del $\mathrm{V}$ libro d'APollonio.

$\left.{ }^{29}\right)$ In una lettera del 30 luglio 1693 , ai redattori del Joumal res Sçavnns, MagdaAbecGi fa cenno della Geometria motus inviatagli in dono da CEva ; cfr. Le Jousmal des Sçrons, pour lamiée M.DC.XCIII. Avec le Catalogue des tivres dont il y est parlé, et une Table des Matieres. Nouvelle edrition (A Paris, chez Witte, M.DCC.XXIX), p. 342. 


\section{Al Sig.: Giovanni Ceva \\ Mantova \\ 3 marzo 1693}

1ll." Sig. mio Sig. Pad. Col.

La sera del Martedi prossimo passato mi pervenne dalla Posta la gentiliss. . . . di V. S. Ill. ${ }^{\text {na }}$ per la quale mi dà avviso d'inviarmi tre copie d'un suo nuovo libro che una per me, l'altra al Sig." Bellini, la 3 al sig..... [Magliabecchi] una per chi in essa non vi si dice.

Se tal missione sia stata gì̀ fatta, ne per qual via, e mi sovvenne che altra volta per ricever da me alcune delle mie opere V.S. Ill." si valse di certi Ebrei a quali mi impose di farne la consegna, mi credei ancora che di un mezzo simile ella si fosse valsa adesso per farmi avere le sudd. copie: ond'io m'ero accomodato prima di rispondere di attender per tal via la con. segna di quelle quale in breve speravo. Non ostante dentro a questo tempo non ho mancato di intender da questi Ebrei.... et alla stanza di Procacci se per sorta vi fosse questo involto, ma non già alla Posta di dove un mio Ser." recuperò la lettera, stante che ne men su la coperta di essa era indicata questa missione.

Ma stassera in passando a caso dalla Posta mi venne in pensiero di domandar se per fortuna vi fosse per me qualche involto di libri; et quale di fatto trovai che vi era, e vi averebbe dimorato fin tanto che questi.... non ne fossero ricercati, essendo soliti di attender che mandi a prendergli quel tale che possa averue avuto lettera d'avviso, la quale non era annessa all'involto. Subito dunque ne mandai uno a legare et in questo giomo l'ho scorso tutto con estrema aviditò, e così alla sfuggita ho compreso esser un degno parto del di lei fertilissimo ingegno e non quale l'innata modestia sua pretendeva di figurarmelo.

Ben è ver che di questa mia età di 71 anni agravata da tanti mali non ho più la memoria così tenace nè la mente così chiara . . . . . . . . . . . ......... si che io non [?] mi faccio sicuro di poter continuare a studiar quest'opera sua come bramerei, e avrei fatto di mia gioventì. 
Nondimeno rendo umil. grazie alla S. V. Ill." della memoria che ella conserva di me così cadente ch'io sono, col tenermi nel numero di quelli che ne sien capaci . . . . . . . . . . . . . . . . . . . . . .

Ho già mandato in nome di lei un esemplare per ciascuno a suddetti Signori da quali doverà sentirne il recapito, e poichè la sua liberalità si è estesa invece di tre di mandarne quattro ad un giovane mio Nipote che ha qualche ..... e diletto in queste materie, ed in nome di questo ancora le rendo vivissime grazie.

Fra non molto spero di mandarle qualcosa di mio, se Dio lo permetterà, e fra queste una Leltera scritla ad un nostro comune Amico, con altresì appresso dei miei studi piu vecchi dove alcune delle cose che mi par di vedere in questa sua nobiliss. ${ }^{m a}$ opera, le vedrà trattate diversam. ${ }^{+e}$

Vedo qui che ella ha fatto assai piu conto di quel che si meritava quella mia bagatella sopra il moto de pendoli col riferirla nel proprio libro, dandole così quel pregio che per se stessa non ha; ma però in comparazione delle sue profonde e solide speculazioni spiccherà molto più la debolezza di quella alla quale 'io gì̀ non pensava più.

Ben è vero che quegli onorati attributi ch'ella si è compiaciuta di darmi non si devono ad uno che sà d'esser inferior di merito a qualsisia ben novizio scolare e che in tempo di vita sua è stato occupato sempre in affari pubblici e del suo Sig." . . . . operando cose alienissime a quelle che nella somma quiete si meditano al tavolino . . . . onde chi ben mi conosce dirà ch'ella si sia lasciata trasportar dall'affetto ch'ella mi porta, e di questo io mi pregio.....

Per adesso invio alla S. V. Ill." due esemplari d'un mio libercolo stampato mesi sono per comando di chi sopra di me ha sovrana autorità. Uno servirà per lei, e l'altro per chi più le aggrada; e tutto riceverà franco per la posta con questa mia. Compatisca se una materia così grave e mera speculazione geom." io l'ho portata come in ischerzo a meccanica per via ditimpano e di suono, che così fare m'è convenuto per dar nel genio; . . . . . . .

Riderebbe V. S. in veder le strane risposte che son comparse da diversi, e da fa. ${ }^{\text {mi }}$ Geometri e Analitici d'Europa nel propos. del . . . . Problema non ostante che nel libretto io abbia accennato tanto che basta per ricavar la soluzione e dimostraz." e tardi mi son avvisto che io doveva scrivere in latino, e non in toscano per farlo comune a tutti, e a quei che han preso scusa di non intender questa lingua.

Ma ora io non mi sento da far di nuovo una simile fatica: la quale, se 
Dio vorrò, si vedrà portata per la sua via e inclusa in altra mia opera antica elementare.

E qui per non più tediar chi si gloriosanente operando spende il suo tempo, con riverente ossequio mi confermo

Di V. S. Ill."

Di Fir. 3 Marzo

1692

V. V.

Il libercolo che Viviani dice di iuviare a Geva deve esser certamente quello che conteneva la risoluzione del famoso problema ch'egli propose negli Acta Eruditorum, e che oggi va appunto sotto il nome di problema di Viviani $\left({ }^{30}\right)$ (aprire quattro finestre in una volta enisferica, in modo che la rimanente parte di questa sia quadrabile).

L'enigma fu risolto, come si sa, analiticamente da molti dei maggiori matematici dell'epoca (Leibniz, Giacomo Bennoulli, Wallis, de l'Hospital), e di ciò Viviani rimase molto addolorato, giacchè aveva così avuta una prova. dell'eccellenza di quei metodi analitici, ch'egli reputava inferiori ed inadatti alla miglior risoluzione dei problemi geometrici.

Nella copia riprodotta della lettera egli chiama strane le risposte date al suo problema; e si duole, in sostanza, che gli altri matematici non avessero risoluto il problema coi metodi suoi $\left({ }^{31}\right)$. Egli, nella sua risoluzione, non inserì le dimostrazioni; queste, peraltro, furon poi date da Gurdo Grandi nella

$\left({ }^{30}\right)$ Vivianı pose il suo enigma col titolo. di Aenigma geometricum de miro opificio Testudinis quadrabilis emisphericae a D. Pio Lisci pupillo geometra propositum, die 4 Aprilis A. 1692 (Acta Eruditorum, a. 1692, p. 274), nascondendo così il suo nome con l'anagramma dell'appellativo postremo Galilaei discipulo. Ne diede la soluzione in Al Serenissimo Principe di Toscana, Formazione, e misura di tutti $i$ cieli, con la struttura, e quadratura esatta dell'intero, e delle parti di un nuovo Cielo ammirabile, e di uno degli antichi delle volte regolari degli architetti. Curiosa esercitazione matematica di V. V. (In Firenze, nella st. di Piero Matini, 1692).

(11) Il Montucla, nella sua Histoire des mathématiques, dans laquelle ond rend compte de leurs progrès depuis leur origine jusqu'à nos jours; etc. Tome II (Paris, chez Jombert, M.DCC.LVIII), p. 71, è di opinione che nessuna delle soluzioni date del problema, superi per eleganza quella data dal Vivian. 
sua prima pubblicazione matematica $\left({ }^{32}\right)$. Notiamo anzi che Grand diede poi anche la traduzione latina dell'opera di Viviani, traduzione che Viviani nella lettera precedente si rammarica di non aver pensato di stampare.

G. CEva rispose allora coll'altrà seguente lettera:

$$
\text { Ill. }{ }^{m o} \text { Sig. mio Pne Col. }{ }^{m *}
$$

Non fui veramente avvertito di avvisarla, che le quattro coppie del mio libro, gliele trasmettevo con il corriere, ben mi avviddi quasi subb..$^{\circ}$ partito il Corriere med., ma non servì; men male che V. S. Ill." se l'immaginò, e con ciò hebbi adempito il mio desiderio, che ella restasse prontam." servita, come che anche questo concorreva alla gran stima che si deve al merito di V. S. Ill." Quanto poi alli meriti del libro, ella che n'è quel gran Giudice sạprà quello possa pesare, e stimo che l'affetto . . . . pure comunque sia ho inteso d'imprimere in quella le note dell'alto ossequio, che le proffesso. Se io havessi letto prima il bellissimo suo libro de maximis et minimis, havrei facilitate molte dimostrazioni; massime quelle, che caminano per doppia posizione, bastandone di una, ma servirammi in avvenire, e così potrò gloriarmi d'essere vero scolare di V.S. Ill." alla quale vorrei crescere una buona parte di vita, e di prosperità per godere lungo tempo un tanto mio Padrone, ma son questi desideri mere vanità, e la veritò̀ è che habbiamo a godere un Paese di gran lunga più bello se Iddio ci farà la grazia per sua misericordia.

La ringrazio del ricapito delli due esemplari, e che abbia collocato cosi bene anco il quarto, godendo che un suo P. Nipote camini per una strada così feconda per grandi cose, massime guidato dall'ingegno grande di V. S. Ill." ${ }^{\text {ma }}$, e di $q^{e{ }^{t h o}}$ che esso havrà dalla propria indole.

Mi sarà sopramodo caro di vedere l'allro libro, che in breve pensa V. S. Ill." di collocare in luce, e non potrò essere che degno delle sue bellissime idee, si come lo è il libricino che ho ricevuto, e visto con avvidità, nell'ordinario stesso, e non (so) se non scorgere in V. S. Ill." argomenti della gran sua bontà, ingegno, e generosità, e quanto a questo ultino suo libro mi riserbo a goderlo piu diffusam. ${ }^{\text {te }}$ havendo hora qualche occupazione, che non mi lascia l'animo del tutto libero.

${ }^{\left({ }^{82}\right)}$ G. GRANDI, Geometrica demonstratio Vivianeorum problematum quae in exercitatione yeometrica, etrusco idionate anno 1692 edita, cum sola determinatione prodidierant, ecc. (Flom rentiae, ex typ. de Guiduccis, M.DC.IC). 
Quella mia machinetta, o sia balestra $\left({ }^{3}\right)$ l'ho fatta far qui et è stata molto gradita da questo ser. ${ }^{\text {mo }}$ mio Prne, al quale ho dedicato il libro. L'artefice è un tal francese detto Monsì Cotel $\left({ }^{34}\right)$, ̀̀ questo un artefice, che credo habbia pochissimi pari, poichè oltre il maneggiare bene il martello, e lima, lavora anco d'ingegno con belle inventioni della sua arte. La sua eccellenza ha meritato di esser già molto tempo ..... da Sua Altezza, e se V. S. Ill." comanderà che sia servito codesto Ser." Prencipe mi prometto di una cosa da par suo quanto al lavoriero, e per quello riguarda la sostanza della macchina (benchè è stato mio ardire il crederla degno di un Prencipe) tutta volta contribuirò ogni mia assistenza, acciochè sia di gran lunga più efficace della. già fatta.

Circa il connercio epistolico tengo quello del Valisio, che V. S. Ill. ${ }^{\text {sa }}$ con la sua solita generosità mi regalò insieme cole sue opere già anni sono, e se mai a caso fosse questo, che la ricercasse me lo accenni, che subb." glielo invierò. Da questo hebbi luce esservi stampato di d." Vallisio altro libro inscritto arithmetica infinitorum, e per quante diligenze io habbia usate non l'ho mai potuto rinvenire come desideravo, perchè tratta di methodo molto bello, e qui con ogni più riverente ossequio resto $d i$ V. S. Ill. ma $^{\text {ma }}$

Mant. "li 13 marzo 1693.

Devot." ed obligat. Serv.

Giovanni Geva.

11. Colla precedente lettera finisce il carteggio Ceva-Viviani conservatoci, della fine del ' 600 .

Gi rimane ancora una lettera del Ceva del 1701:

$$
\text { Ill. }{ }^{m o} \text { Sig. }{ }^{.} \text {Prne Col. }{ }^{m o}
$$

Haverà V. S. Ill." "a tempo fa ricevuto dal Pre Tomaso nio fr. ${ }^{\text {elto }}$ un di lui libro nel quale evvi un trattatello de natura gravium $\left({ }^{35}\right)$, e come che tra le

$\left.{ }^{(33}\right)$ Questa balestra è quella descritta in fine della Geometria motus.

(34) Questo artefice è quello che, secondo ogni probabilità, costrù l'apparecchio ideato da Tommaso Geva per la plurisezione dell'angolo. Cír. A. Pascal, Liapparecchio polisettore di Tommaso Ceva ed una lettera inedita di Guido Grandi (Rendiconti del R. Istituto Lombardo di Scienze e Lettere, vol. XLVIII, 1915).

(5) Th. Cevae, De natura gravium Libri duo (Mediolani, typ. Malatestae, 1699). 
altre cose, alle quali non sapevo piegare il mio assenso, eravi, che $i$ gravi dovessero in proporzion delle distanze dal centro dell'universo aquistare mass. velocità, nè quel suo methodo parevami efficace per dimostrare una cosa tanto lontana dal senso comune, gli riuscì per via di movimenti, che si consumano nel med. tempo scorrendo il grave realm. ${ }^{e}$ per un lato d'un triangolo, e virtualm." per gli altri duoi, farmi col beneficio di una libra immaginaria, render per qualche tempo persuaso; ma riandando poscia la sua argumentat. ${ }^{n e}$, mi nacquero dificoltà tali, che mi fecero ritornare nella primiera mia, e vulgata opinione, che de gravi la distanza dal centro nulla conferisse al supposto augmento di velocità; per lo che pensando di poter in esso suo argomento ritrovare una manifesta fallaccia, fui io per il contrario da me med." convinto, con la dimostr., seguente; ma poichè può essere, che prenda qualche equivoco, venendomi questa messa in dubbio da un Pre della Compagnia di Gesì a cui l'ho comunicata, siamo convenuli di porla sotto la giustiss ${ }^{u}$ censura di V.S. Ill. ${ }^{m a}$, la quale, come amica della verità, e peritissimo di tali scienze, non vorrò dire, che puramente il suo sentimento.

Siano dunque $i$ duoi corpi gravi $A D, C B$, i centri dei quali $A, B ; B E$, $A E$ siano le distanze loro dal centro $E$ dell'universo: dico che la velocità di $B C$ dalla quiete in $B$ alla velocità di $D A$ dalla quiete in $A$ verso il medesimo centro $E$ sta come la distanza $B E$ quanta si vogli alla distanza $A E$; sia $F$ il centro di gravezza de' duoi corpi gravi $A D, B C$; si che supposta

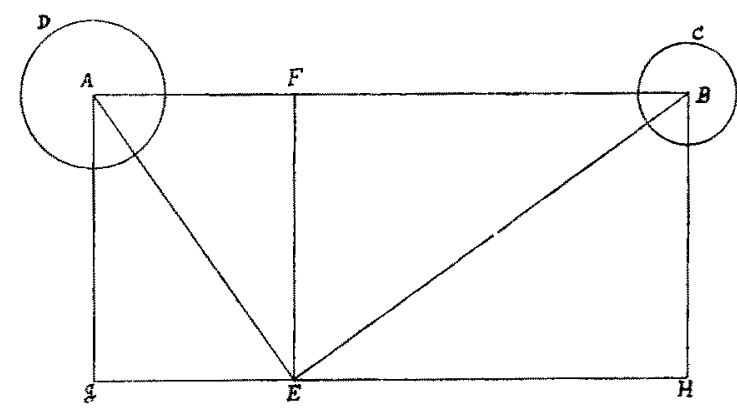

Fig. IV. $A F B$ una libra, equiponderi $q^{\text {esta }}$ sospesa dal punto F. Tirisi poi per E la $G E H$ parallela alla $A F B$, e parimenti da $B$, et $A$ le due $B H, A G$ parallele alla $F E$, la quale per più facilitì intendasi perpendicolare alla $A B$. Poichè supposta $B E$ la velocità del grave $C B$ dalla quiete in $B$ equivale la med." alle due $B F$, $B H$, sarà egualm. tirate l'estremo

$B$ dal grave $B C$ giusta la direttione $B E$, che dal corpo $B C$ considerato senza gravezza, ma affetto co le due velocità $B H, B F$ : dunque il grave $B C$ farà forza secondo la direttione $B H$ con la velocità $B H$, e secondo $B F$ con la velocità $B F, e$ poichè si suppone la libra $A B$ in equilibrio slarò $B F$ ad $A F$ come il corpo $A D$ al corpo $B C$, ciò̀ il corpo $D A$ dovrà havere la medesima 
velocità secondo $A G$, che $C B$ secondo la $B H$, ciò̀ essendo $B H$ la velocità di $B C$ sarà $A G$ quella di $D A$; e poichè per il med. equilibrio il momento del grave $B C$ verso $A$ è uguale al momento del grave $D A$ verso $B$, sarà la velocità del corpo $B C$ a quella di $D A$, verso $F$, come il corpo $D A$ al corpo $C B$, ciò̀ come la $B F$ ad $A F$; ma la velocità di $B C$ verso $F$ fa $B F$, dunque quella di $D A$ verso $F$ sarà $A F$; tanto che il corpo affetto delle due velocità $A F, A G$ sarà equilibrato con il grave $B C$, osia con il corpo affetto delle due velocità $B H, B F$; ma poichè il corpo $D A$ affetto delle velocità $A F, A G$ equivale al grave $D A$ (sendo che tanto l'uno, quanto l'altro equipondera al contraposto $C B$ ) dunque il grave $D A$ haverà in $A$ la velocità $A E$, che è equivalente alle due .... laonde il corpo $C B$ in $B$ dalla quiete haverà una tal velocità, che a quella di $D A$ in $A$ (ambi verso $E$ ) starà come $B E$ ad $A E$, che ̀̀ quello etc.

Corollario.

Dalla sudetta dimostraz." si vede, che la libra $A B$, il di cui centro in $F$, intanto sta in equilibrio in quanto la resta $B F$ alla $F A$ sta come la mole corporea $D A$ alla mole corporea $C B$; e non già in proporzion reciproca de pesi, come volgarm. ${ }^{e} \dot{e}$ ricreduto.

Corollario.

Parimenti per la similitudine de movimenti, e che si consummano in uguali tempi secondo la $B H$, et $A G$; facilm. si dimostra, che $i$ tempi per le $B E$, $A E$ sarebbero uguali.

Scholio.

Si è proposta una figura facile per abbreviare la dimostrazione; per altro in qualsivoglia inclinazione di libra, si dimostra lo stesso; indi poi un harmonia maravigliosa, con la quale $i$ gravi cadendo nello stesso tempo verso 
il centro dell'universo, concorrono anco a particolari; ciò̀ in questo caso in $E$, et $F$.

Compatisca V. S. Ill. ${ }^{\text {anc }}$ il disturbo, ma gia chè si tratta di cosa assai curiosa, non ho temuto di prendermi questa libertà, e qui con tutto l'ossequio resto con inchinarla.

Di V. S. Ill. ${ }^{m a}$, la quale supplico darmi pure qualche nuova del S. D. Lorenzo Bellini mio riverit." Prne, al quale pure potrà se gli piace, communicare essa dimostrazione, sperando di poter poi dire come nasca la med. ${ }^{a}$ gravezza. Mantua li 11 Marzo 1701.

Devot." et obligat." Serv."

Giovanni Geva.

Napoli, Facoltà Matematica della R. Università, marzo 191 ó. 